EPJ Web of Conferences 112,01022 (2016)

DOI: $10.1051 /$ epiconf/201611201022

(C) Owned by the authors, published by EDP Sciences, 2016

\title{
Electron-deuteron DIS with spectator tagging at EIC: Development of theoretical framework
}

\author{
W. Cosyn ${ }^{1}$, V. Guzey ${ }^{2}$, M. Sargsian ${ }^{3}$, M. Strikman ${ }^{4}$, and C. Weiss ${ }^{5}$ \\ ${ }^{1}$ Ghent University, 9000 Ghent, Belgium \\ ${ }^{2}$ Petersburg Nuclear Physics Institute, Gatchina, 188300, Russia \\ ${ }^{3}$ Florida International University, Miami, FL 33199, USA \\ ${ }^{4}$ Pennsylvania State University, University Park, PA 16802, USA \\ ${ }^{5}$ Jefferson Lab, Newport News, VA 23606, USA
}

\begin{abstract}
An Electron-Ion Collider (EIC) would enable next-generation measurements of deep-inelastic scattering (DIS) on the deuteron with detection of a forward-moving nucleon $(p, n)$ and measurement of its recoil momentum ("spectator tagging"). Such experiments offer full control of the nuclear configuration during the high-energy process and can be used for precision studies of the neutron's partonic structure and its spin dependence, nuclear modifications of partonic structure, and nuclear shadowing at small $x$. We review the theoretical description of spectator tagging at EIC energies (lightfront nuclear structure, on-shell extrapolation in the recoil nucleon momentum, final-state interactions, diffractive effects at small $x$ ) and report about on-going developments.
\end{abstract}

Measurements of deep-inelastic scattering and related processes on light ions $(A=2,3, \ldots)$ address basic questions of high-energy nuclear physics and Quantum Chromodynamics (QCD), such as the partonic structure of the neutron and its spin decomposition [1], the nuclear modification of the nucleon's quark and gluon densities [2,3], and the onset of nuclear shadowing and coherent phenomena at small $x$ [4]. The common challenge in the analysis of such measurements is to account for the multitude of nuclear configurations present during the high-energy scattering process. The scattering can involve any of the constituent nucleons $(p, n)$ in different states of their quantum-mechanical motion (momentum, spin), as well as non-nucleonic degrees of freedom excited by the nuclear binding. In the extraction of neutron structure one needs to isolate the cross section arising from scattering on the neutrons and eliminate the effects of nuclear binding (Fermi motion, non-nucleonic degrees of freedom). For neutron spin structure one must also infer the effective polarization of the neutron and account for the polarization of non-nucleonic degrees of freedom, particularly intrinsic $\Delta$ isobars in polarized ${ }^{3} \mathrm{He}$ [5]. In the study of nuclear modifications at $x \gtrsim 0.1$ (EMC effect, antishadowing) one would like to relate the modifications to the interactions taking place in a particular configuration (exchange mechanisms, short-range correlations). In the analysis of coherent phenomena at small $x$ one wants to understand what configurations in the wave function build up the coherent response (onset of shadowing, saturation). While these challenges can partly be addressed by theoretical calculations, major progress could come from new experiments that provide information on the nuclear configurations during the high-energy process.

Deuteron and spectator tagging. High-energy scattering on the deuteron $(A=2)$ with detection of a proton or neutron in the nuclear fragmentation region (recoil momentum $p_{R} \sim$ few $10-100 \mathrm{MeV} / c$ 
in the deuteron rest frame) represents a unique method for performing DIS measurements in controlled nuclear configurations. The deuteron's $p n$ wave function is simple and well known from low-energy measurements, including the light-front wave function entering in high-energy processes (see below). Because the deuteron has isospin $I=0, \Delta$ isobars in the wave function are strongly suppressed (they can occur only in $\Delta \Delta$ configurations), so that the deuteron can be treated as a $p n$ system for most of the configurations relevant to DIS [6]. Detection of the "spectator" nucleon and measurement of its momentum positively identifies the active nucleon and controls its momentum during the DIS process. Because of the simple geometry of high-energy scattering on the $A=2$ system the possibilities for final-state interactions are limited, and in configurations where they occur they can be computed using theoretical models. Regarding polarization the deuteron has $\operatorname{spin} S=1$; its wave function is predominantly S-wave with a small admixture of D-wave, so that the effective nucleon polarization is well known.

DIS on the deuteron with recoil proton detection ("spectator tagging") was measured in the JLab CLAS BONuS experiment at $6 \mathrm{GeV}$ beam energy at recoil momenta $p_{R} \gtrsim 70 \mathrm{MeV} / c$ [7] and will be explored further at $11 \mathrm{GeV}$. In such fixed-target experiments it is difficult to get the slow proton (or neutron) out of the target and measure its momentum with sufficient resolution. Much more suitable for this purpose are colliding-beam experiments, where the spectator nucleon moves on with approximately half the deuteron beam momentum and can be detected using forward detectors. An EIC with electron-nucleon center-of-mass energies $\sqrt{ } s_{e N} \sim 15-100 \mathrm{GeV}$ and luminosity $L \sim 10^{33}$ $10^{34} \mathrm{~cm}^{-2} \mathrm{~s}^{-1}$ is projected as a future facility for nuclear physics [8]. Both EIC designs presently developed have capabilities for forward nucleon detection [9]. The JLab EIC detector is designed to provide full coverage for spectator protons down to zero transverse momentum, and with a momentum resolution corresponding to $p_{R} \sim 20 \mathrm{MeV} / c$ in the rest frame, as well as forward neutron detection [10]. This setup would enable detailed measurements of DIS with spectator tagging over the entire $\left(x, Q^{2}\right)$ range covered by the collider, which includes the region of sea quarks, gluons, and small- $x$ phenomena. It would allow also tagged measurements on the polarized deuteron, which is potentially the most precise method for determining neutron spin structure. Altogether this setup would permit nuclear DIS measurements with full control of the nuclear configuration and enable a new level of understanding of nuclear effects in QCD.

To realize this potential it is necessary to develop the theoretical apparatus for describing DIS with spectator tagging at collider energies and with polarized beams. In this article we summarize the theoretical description and report about on-going developments [11]. A general review of nuclear physics with EIC is given in Ref. [12].

Cross section and structure functions. The basic observable is the invariant differential cross section for inclusive DIS on the deuteron with an identified nucleon with recoil momentum $p_{R}$ in the final state (see Fig. 1a):

$$
\frac{d \sigma\left(e D \rightarrow e^{\prime} N X\right)}{d x d Q^{2}\left(d^{3} p_{R} / E_{R}\right)}=[\text { flux }]\left[F_{2 D}\left(x, Q^{2} ; \alpha_{R}, p_{R T}\right)-(1-\epsilon) F_{L D}(. .)+\ldots\right],
$$

where $\epsilon$ is the virtual photon polarization parameter. The "tagged" deuteron structure functions $F_{2 D}$ and $F_{L D}$ depend on $x$ and $Q^{2}$ as well as on the recoil nucleon momentum, which is parametrized here by the light-cone fraction $\alpha_{R} \equiv 2\left(E_{R}+p_{R}^{z}\right) /\left(E_{D}+p_{D}^{z}\right)$ and transverse momentum $p_{R T} \equiv\left|\boldsymbol{p}_{R T}\right|$ in a frame where the momentum transfer $\boldsymbol{q}$ and the deuteron momentum $\boldsymbol{p}_{D}$ are collinear and along the $z$-axis. Here $x \equiv Q^{2} /\left(p_{D} q\right)=2 x_{\mathrm{B}}$ is the rescaled Bjorken variable with limits $0<x<2$. The ellipsis in Eq. (1) stands for azimuthal angle- $\left(\phi_{R^{-}}\right)$and spin-dependent terms which we left out for brevity. The full form of the cross section for scattering of a polarized electron on a polarized deuteron (vector, tensor) has been determined and will be given elsewhere [13]. We emphasize that the form of Eq. (1) is general and does not rely on any assumptions regarding composite structure of the deuteron. 


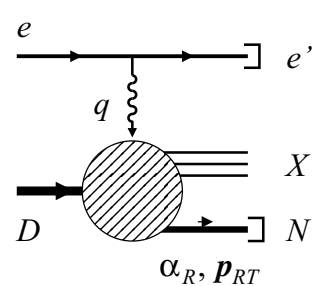

(a)

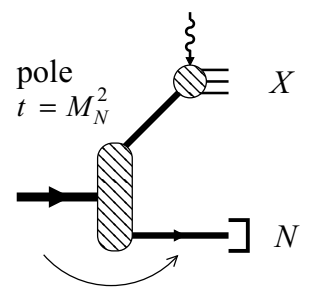

(b) $\quad t=\left(p_{R}-p_{D}\right)^{2}$

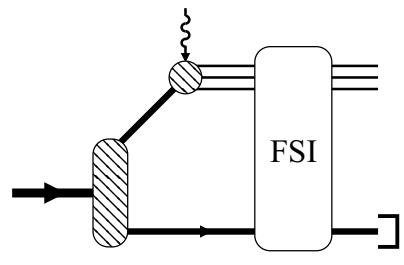

(c)

Figure 1. (a) Tagged DIS on the deuteron, $e D \rightarrow e^{\prime}+N+X$. (b) Impulse approximation amplitude, containing the nucleon pole at $t=M_{N}^{2}$. (c) Final-state interaction amplitude.

Light-front nuclear structure and impulse approximation. The theoretical description aims to express the tagged structure functions of Eq. (1) in terms of nuclear structure and nucleon structure functions. High-energy processes such as DIS probe the nucleus at fixed light-front time, where the structure is described by the light-front wave function. The light-front description is unique in the sense that the off-shellness of the constituents remains finite as the scattering energy becomes large, which permits a composite description of the nucleus in DIS in terms of nucleon degrees of freedom [6]. It can be implemented using either non-covariant light-front quantization or a covariant scheme based on Feynman diagrams [14]. The starting point is the impulse approximation, in which the electromagnetic current interacts with a single nucleon and the DIS final state evolves independently of the other nucleon (see Fig. 1b). Neglecting terms (mass) ${ }^{2} / Q^{2}$ it gives

$$
F_{2 D}\left(x, Q^{2} ; \alpha_{R}, p_{R T}\right)=\frac{\left|\Psi_{D}\left(\alpha_{R}, \boldsymbol{p}_{R T}\right)\right|^{2}}{2-\alpha_{R}} F_{2 N}\left(\widetilde{x}, Q^{2} ; \text { off-shell }\right), \quad \widetilde{x} \equiv \frac{x}{2-\alpha_{R}} .
$$

Here $2-\alpha_{R}$ is the LF momentum fraction of the active nucleon in the deuteron, which is kinematically fixed by that of the spectator, $\alpha_{R}$. The nucleon structure function is evaluated at $\widetilde{x}=x /\left(2-\alpha_{R}\right)$. $\Psi_{D}$ is the deuteron's $N N$ light-front wave function, normalized such that $\int d \alpha_{R} d^{2} p_{R T}\left|\Psi_{D}\left(\alpha_{R}, \boldsymbol{p}_{R T}\right)\right|^{2} /\left[\alpha_{R}(2-\right.$ $\left.\left.\alpha_{R}\right)\right]=1$, which can be obtained from the non-relativistic wave function for rest frame momenta $\left|\boldsymbol{p}_{R}\right| \ll M_{N}$ [6]. $F_{2 N}$ is the structure function of the active nucleon, which is generally modified by off-shell effects (see below). When integrated over the recoil momentum Eq. (2) satisfies the baryon number and light-cone momentum sum rules for the deuteron.

Equation (2) implies Bjorken scaling of the tagged deuteron structure function at $Q^{2} \gg 1 \mathrm{GeV}^{2}$ for fixed $\alpha_{R}$ and $p_{R T}$ (this holds even in the presence of modified nucleon structure and final-state interactions). This feature can be verified experimentally and represents a basic test of the theoretical framework. It represents a special case of the QCD factorization theorem for DIS with identified slow hadrons in the target fragmentation region [16].

Nucleon pole and on-shell extrapolation. An essential feature of the impulse approximation is that it captures the leading singularity of the tagged structure function in the invariant momentum transfer between the deuteron and the recoiling nucleon, $t \equiv\left(p_{D}-p_{R}\right)^{2}=\operatorname{function}\left(\alpha_{R}, p_{R T}\right)$. The deuteron LF wave function has a pole at $t=M_{N}^{2}$ corresponding to the nucleon on-shell point. (In the covariant formulation this pole represents "nucleon exchange" between the deuteron and the electromagnetic current.) In the limit $t \rightarrow M_{N}^{2}$ one therefore has

$$
F_{2 D}\left(x, Q^{2} ; \alpha_{R}, p_{R T}\right) \sim \frac{R}{\left(t-M_{N}^{2}\right)^{2}} F_{2 N}\left(\widetilde{x}, Q^{2}\right) \quad\left(t \rightarrow M_{N}^{2}\right),
$$




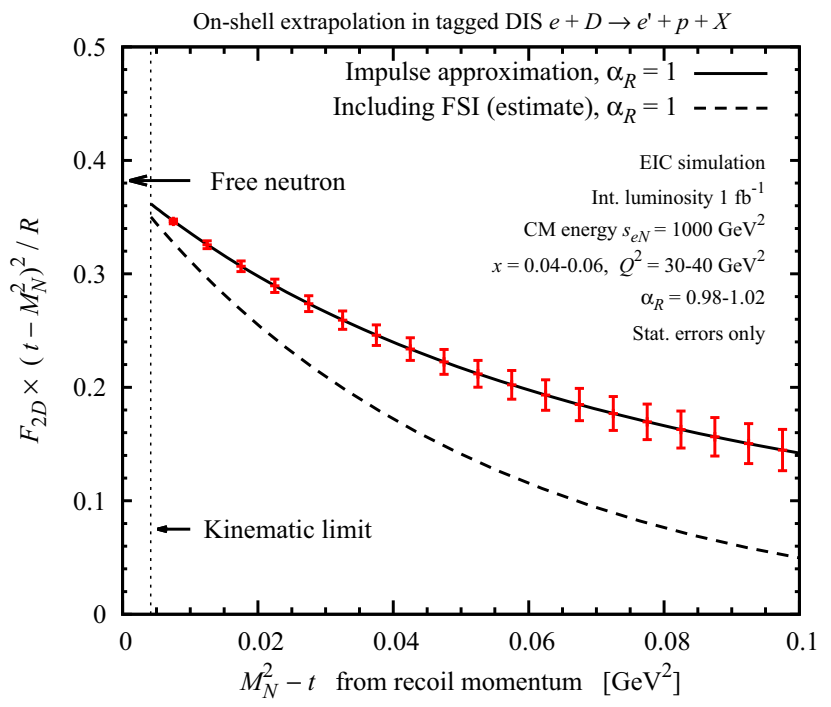

Figure 2. Neutron structure extraction by on-shell extrapolation $t \rightarrow M_{N}^{2}$ in tagged DIS $e+D \rightarrow e^{\prime}+p+X$. The plot shows the tagged deuteron structure function $F_{2 D}$, with the pole factor $R /\left(t-M_{N}^{2}\right)^{2}$ removed, as a function of the off-shellness $t-M_{N}^{2}$, at $\alpha_{R}=1$. Solid line: Impulse approximation. Dashed line: Sum of impulse approximation and final state interactions (estimate) [15]. Data: Simulated measurement of $t$-dependence with EIC. Parameters are shown on the plot. Error bars indicate expected statistical errors; for systematic errors see text.

where $R \equiv R\left(\alpha_{R}\right)$ is a calculable residue and $F_{2 N}$ is the on-shell (free) structure function of the active nucleon. Theoretical arguments show that final-state interactions (see Fig. 1c) do not modify the leading singularity in $t$, as they involve an integral over the intermediate nucleon momentum [17]. One can thus obtain the on-shell nucleon structure function by measuring the deuteron structure function $F_{2 D}$ over a range of $t$ at fixed $\alpha_{R}$ and performing the on-shell extrapolation to $t \rightarrow M_{N}^{2}$. The physical region is $t-M_{N}^{2}<-\epsilon_{D} M_{D}=-0.0041 \mathrm{GeV}^{2}$ ( $\epsilon_{D}$ is the deuteron binding energy), so that measurements can be done extremely close to the on-shell point. In the deuteron rest frame $t-M_{N}^{2}=-2\left|\boldsymbol{p}_{R}\right|^{2}-\epsilon_{D} M_{D}$, and the kinematic limit corresponds to zero recoil momentum $\boldsymbol{p}_{R}=0$.

Simulations show that precise measurements of the free neutron structure function $F_{2 n}$ by proton tagging and on-shell extrapolation are feasible with EIC (see Fig. 2). One measures the tagged deuteron structure function $F_{2 D}$ over a range of $t$ at fixed $\alpha_{R}$, removes the pole factor in Eq. (3), and performs polynomial extrapolation to $t \rightarrow 0$. Both statistical and systematic data errors can be propagated through the extrapolation procedure. Systematic errors arise mainly from the uncertainty in the recoil momentum determination and are expected to result in a correlated $F_{2 n}$ uncertainty of $\lesssim 3 \%$ with the JLab EIC beam and detector design [11].

The on-shell extrapolation can also be used to extract neutron spin structure functions from polarized tagged DIS on the deuteron. It is convenient to measure the double spin asymmetry of the tagged cross section as a function of the recoil momentum,

$$
A_{\|}\left(x, Q^{2} ; \alpha_{R}, p_{R T}\right)=\frac{\sigma\left(+\frac{1}{2},+1\right)-\sigma\left(-\frac{1}{2},+1\right)}{\sigma\left(+\frac{1}{2},+1\right)+\sigma\left(-\frac{1}{2},+1\right)},
$$

and perform the extrapolation to $t \rightarrow M_{N}^{2}$ for the asymmetry rather than the cross sections. [Here $\sigma\left(\lambda_{e}, \lambda_{D}\right)$ denotes the cross section for electron helicity $\lambda_{e}= \pm 1 / 2$ and deuteron helicity $\lambda_{D}= \pm 1$, i.e., longitudinal polarization along the respective beam directions.] The asymmetry exhibits only very weak $t$ dependence. An interesting feature is that the $\mathrm{D}$-wave in the deuteron wave function practically drops out in the on-shell extrapolation, since its wave function is proportional to the squared rest frame nucleon momentum $\left|\boldsymbol{p}_{R}\right|^{2}$ and the on-shell point corresponds to extremely small unphysical momenta 
$\left|\boldsymbol{p}_{R}\right|^{2}=-\epsilon_{D} M_{D}$. This means that at the on-shell point the neutron is effectively $100 \%$ polarized in the deuteron spin direction. Furthermore, kinematic factors and many systematic errors cancel in the cross section ratio. Simulations show that precise measurements of neutron spin structure with on-shell extrapolation are possible over most of the $\left(x, Q^{2}\right)$ range covered by EIC with an integrated luminosity $\sim 100 \mathrm{fb}^{-1}[18]$.

Nuclear modifications and final-state interactions (FSI). Away from the nucleon pole $t=M_{N}^{2}$ the tagged deuteron structure function is modified both by possible off-shell dependence of the nucleon structure functions and by FSI. The off-shell modifications can be accommodated within the impulse approximation, by allowing the effective nucleon structure function to depend on $t-M_{N}^{2}$ in a certain range of virtualities ("virtual nucleon model"). Specific dynamical models for the off-shell dependence have been proposed.

FSI arise from the interactions of the spectator with the DIS final state produced by the active nucleon (see Fig. 1c). While they do not change the total (untagged) DIS cross section on the deuteron, such interactions can distort the recoil momentum spectrum and change the outgoing particle flux, and must therefore be accounted for in the analysis of tagged DIS. FSI can be estimated theoretically by modeling the composition of the nucleon DIS final state and its rescattering from the spectator. Theoretical arguments suggest that at $x \lesssim 0.1$ the dominant FSI arise from "slow" hadrons in the DIS final state, with rest frame momenta $\sim 1-2 \mathrm{GeV} / c$, as these hadrons are expected to form close to the interaction point and can rescatter from the spectator with hadronic cross sections [15]. "Fast" hadrons with momenta $\gg 1 \mathrm{GeV} / c$ form outside the nucleus and interact marginally with the spectators, as evidenced by the absence of absorption of such hadrons in DIS on larger nuclei [19]. Estimates of the FSI effects in tagged DIS can be made in a schematic model, using empirical DIS hadron distributions and rescattering cross sections [15]. Preliminary results show noticeable modifications of the $t$-dependence of the tagged structure function away from the pole (see Fig. 2). The modifications vanish in the limit $t \rightarrow M_{N}^{2}$, in accordance with the findings of Ref. [17], and thus do not prevent the extraction of free neutron structure through on-shell extrapolation; their effect on the extraction errors remains to be investigated. ${ }^{1}$

An interesting question is how the effects of modified nucleon structure and FSI in tagged DIS could be separated experimentally in future measurements at EIC. Tagged DIS measurements at $\widetilde{x} \sim$ 0.2 , where nuclear modifications of the inclusive structure functions are negligible [2], could isolate the effects of FSI in the tagged DIS cross section. Direct evidence for the dominance of FSI in this region could come from observation of their distinctive angular dependence at larger rest frame recoil momenta $p_{R} \gtrsim 200 \mathrm{MeV} / c$ [15]. Tagged DIS measurements at $\widetilde{x}>0.3$, where substantial nuclear modifications are seen in the inclusive data (EMC effect) [2], could then reveal the combined effects of modified nucleon structure and FSI. Further information on nuclear FSI will come from measurements of nuclear modification of hadron spectra in DIS on heavier nuclei. The experimental capabilities of EIC (kinematic range, recoil momentum coverage and resolution, variety of nuclear beams) would enable a program of comparative measurements designed to separate the two effects.

Diffraction in tagged DIS. In tagged DIS at $x \lesssim 0.01$ a new mechanism of FSI arises due to diffractive scattering on the active nucleon, in which the nucleon is left intact and appears in the DIS final state with a rest-frame momentum $\sim$ few $100 \mathrm{MeV} / c$ (see Fig. 3). In DIS on the deuteron such diffractive scattering can happen on the proton as well as on the neutron, with quantum-mechanical interference of the two amplitudes in the cross section. In inclusive DIS $e+D \rightarrow e^{\prime}+X$ this effect

\footnotetext{
${ }^{1}$ The FSI effect on the $t$-distribution depends on the recoil light-cone fraction $\alpha_{R}$ [15]. FSI is maximal for the situation shown in Fig. 2, $\alpha_{R}=1$, which corresponds to an angle of $\sim 90^{\circ}$ between the recoil momentum $\boldsymbol{p}_{R}$ and the $\boldsymbol{q}$-vector in the rest frame (sideways recoil). FSI becomes smaller for $\alpha_{R}<1$ or $>1$, which correspond to angles $<90^{\circ}$ or $>90^{\circ}$ (forward or backward recoil). However, for $\alpha_{R} \neq 1$ the kinematic limit in $t-M_{N}^{2}$ moves further away from zero, which is not favorable for on-shell extrapolation.
} 


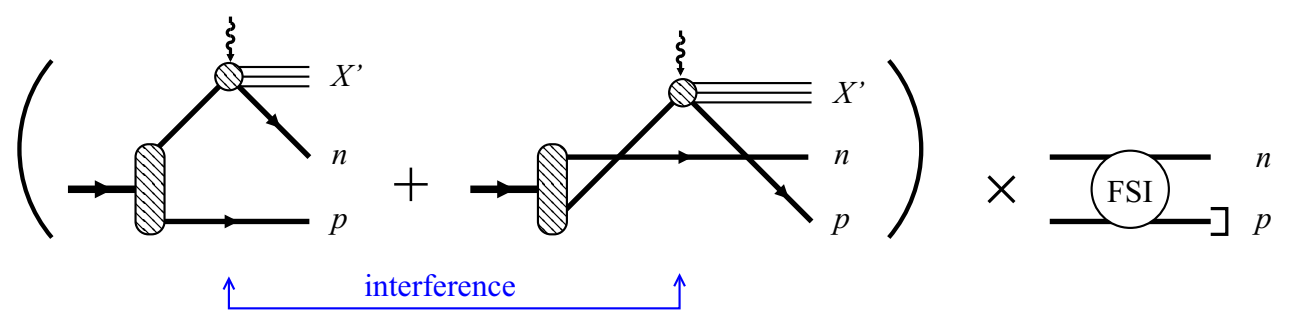

Figure 3. Diffraction in tagged DIS on the deuteron $e+D \rightarrow e^{\prime}+p+X$. Diffractive scattering on the $p$ and $n$ enables interference of the respective amplitudes (shadowing), as well as strong FSI within the outgoing $p n$ system.

gives rise to nuclear shadowing and has been the subject of extensive theoretical studies [4]. In tagged DIS $e+D \rightarrow e^{\prime}+N+X$ the presence of the slow diffractive nucleon in the final state enables strong FSI between that nucleon and the spectator, resulting in considerable modification of the recoil momentum distribution compared to the impulse approximation. The distortion is particularly strong in the spin1 , isospin-0 partial wave of the $p n$ system, where the outgoing $p n$ scattering state must be orthogonal to the deuteron bound state in that channel (a similar effect happens in low-energy deuteron breakup reactions). Theoretical work aims to calculate this effect using methods analogous to those used for shadowing in inclusive DIS [20].

Experiments at EIC could perform detailed tests of the predicted distortion effects using singlenucleon tagging $e+D \rightarrow e^{\prime}+p+X$, or double-nucleon tagging $e+D \rightarrow e^{\prime}+p+n+X^{\prime}$, which completely fixes the kinematics of the produced $p n$ system. In this way they could elucidate the general mechanism of nuclear shadowing, which is essential for analyzing inclusive scattering on the deuteron (including polarization) and quantifying the approach to saturation at small $x$.

In summary, electron-deuteron DIS with spectator tagging at EIC would enable next-generation measurements of neutron spin structure, nuclear modifications of partonic structure, and small- $x$ shadowing, with maximum control of the nuclear configurations participating in the high-energy process. Theoretical methods based on light-front nuclear structure and analytic properties provide a transparent and efficient description of such measurements, consistent with that of inclusive nuclear DIS. Work is in progress to adapt these methods to collider kinematics and polarized beams.

Notice: Authored by Jefferson Science Associates, LLC under U.S. DOE Contract No. DE-AC0506OR23177. The U.S. Government retains a non-exclusive, paid-up, irrevocable, world-wide license to publish or reproduce this manuscript for U.S. Government purposes.

\section{References}

[1] C. A. Aidala, S. D. Bass, D. Hasch and G. K. Mallot, Rev. Mod. Phys. 85, 655 (2013).

[2] S. Malace, D. Gaskell, D. Higinbotham and I. Cloet, Int. J. Mod. Phys. E 23, 1430013 (2014).

[3] L. Frankfurt and M. Strikman, Phys. Rept. 160, 235 (1988).

[4] L. Frankfurt, V. Guzey and M. Strikman, Phys. Rept. 512, 255 (2012).

[5] L. Frankfurt, V. Guzey and M. Strikman, Phys. Lett. B 381, 379 (1996); F. Bissey, V. Guzey, M. Strikman and A. Thomas, Phys. Rev. C 65, 064317 (2002); C. Boros, V. Guzey, M. Strikman and A. Thomas, Phys. Rev. D 64, 014025 (2001).

[6] L. Frankfurt and M. Strikman, Phys. Rept. 76, 215 (1981).

[7] S. Tkachenko et al. [CLAS Collaboration], Phys. Rev. C 89, 045206 (2014). 
[8] U.S. Department of Energy Office of Science Nuclear Science Advisory Committee (NSAC) 2015 Long Range Plan for Nuclear Science, http://science . energy . gov/np/nsac

[9] For current information on the EIC machine designs, see: https://eic.jlab.org/wiki/(JLab) and https://wiki.bnl.gov/eic/ (BNL).

[10] P. Nadel-Turonski, Presentation at Electron-Ion User Group Meeting 2016, UC Berkeley, 6-9 January 2016, https://conferences . lbl .gov/event/56/

[11] C. Weiss et al., "Physics potential of polarized light ions with EIC@JLab," Jefferson Lab 2014/15 Laboratory-directed R\&D Project, https: //www . jlab.org/theory/tag/

[12] A. Accardi, V. Guzey, A. Prokudin and C. Weiss, Eur. Phys. J. A 48, 92 (2012).

[13] W. Cosyn, M. Sargsian, C. Weiss, in preparation.

[14] M. Sargsian, Int. J. Mod. Phys. E 10, 405 (2001)

[15] M. Strikman and C. Weiss, in preparation.

[16] L. Trentadue and G. Veneziano, Phys. Lett. B 323, 201 (1994); J. C. Collins, Phys. Rev. D 57, 3051 (1998) [Erratum-ibid. D 61, 019902 (2000)].

[17] M. Sargsian and M. Strikman, Phys. Lett. B 639, 223 (2006).

[18] W. Cosyn et al., J. Phys. Conf. Ser. 543, 012007 (2014).

[19] J. Ashman et al. [European Muon Collaboration], Z. Phys. C 52, 1 (1991); A. Airapetian et al. [HERMES Collaboration], Eur. Phys. J. A 47, 113 (2011).

[20] V. Guzey et al., in preparation. 